\title{
$S$-dual amplitude and $\mathrm{D}_{3}$-brane couplings
}

\author{
Komeil Babaei Velni ${ }^{1, \dagger}$ and H Babaei-Aghbolagh ${ }^{2, *}$ \\ ${ }^{1}$ Department of Physics, University of Guilan, P.O. Box 41335-1914, Rasht, Iran \\ ${ }^{2}$ Department of Physics, University of Mohaghegh Ardabili, P.O. Box 179, Ardabil, Iran
}

(Received 6 January 2019; published 15 March 2019)

\begin{abstract}
Recently, it has been observed that the $I I B$ scattering amplitudes are compatible with the standard rules of $S$-duality. Inspired by this observation, we will find the tree-level $S$-matrix elements of one RamondRamond and three open strings by imposing this symmetry on the tree-level $S$-matrix elements of one KalbRamond and three open strings. We also find a $S L(2, R)$ invariant form of the $\mathrm{D}_{3}$-brane effective action containing four gauge fields with derivative corrections that was derived from one-loop level four-point amplitude. Using the expansion of the nonlinear $S L(2, R)$ invariant structures, we find the action with derivative corrections at the level of more gauge fields.
\end{abstract}

DOI: 10.1103/PhysRevD.99.066007

\section{INTRODUCTION}

It has been shown that the consistency of an electrodynamics theory under the duality transformation can be expressed as a requirement in which the Lagrangian must transform under duality in a particular way, defined by the Noether-Gaillard-Zumino (NGZ) identity [1]. So, in general, a nonlinear electrodynamics theory that saturates the NGZ identity is a self-dual theory. One of the familiar selfdual electrodynamics theories is the Born-Infeld theory $\mathcal{L}_{B I}=\sqrt{-\operatorname{det}\left(\eta_{a b}+F_{a b}\right)}-1$, where $F_{a b}=\partial_{a} A_{b}-\partial_{b} A_{a}$ is gauge field strength and $A$ is polarization of the gauge field.

This theory could be enjoyed the $S L(2, R) S$-duality (relevant to string theory which insinuates a strong-weak coupling duality of such theories ${ }^{1}$ ) after taking axion and dilaton fields into account. This action is not invariant under the $S$-duality; however, its equations of motion and energy-momentum tensor are invariant under the $S$-duality as it was shown for the electric-magnetic duality $[3,4]$.

\footnotetext{
*Corresponding author.

h.babaei@uma.ac.ir

babaeivelni@guilan.ac.ir

${ }^{1}$ From the presence of two two-form gauge fields $B_{2}$ (NSNS two-form) and $C_{2}$ (RR two-form) in the string theory, a string can carry two types of charges. These two-forms form a doublet of $S L(2, R)$; it follows that the string also transforms as a doublet. On the other hand, the transformation of the complex field $\tau=$ $C_{0}+i e^{\phi_{0}} \rightarrow-1 / \tau$ evaluated at $C_{0}=0$, changes the sign of the dilaton, which implies that the string coupling constant maps to its inverse [2].

Published by the American Physical Society under the terms of the Creative Commons Attribution 4.0 International license. Further distribution of this work must maintain attribution to the author(s) and the published article's title, journal citation, and DOI. Funded by SCOAP ${ }^{3}$.
}

The supersymmetric p-branes that are supported by Ramond-Ramond $(R R)$ sources are the solutions of type II superstring theories. These branes have alternative descriptions in terms of open strings with Dirichlet boundary conditions. D-branes are described by an effective Dirac-Born-Infeld $(D B I)$ action (supplemented with extra couplings to $R R$ fields) which is closely connected to the Born-Infeld $(B I)$ type effective action of open string theory. We consider the $\mathrm{D}_{3}$-brane of type $I I B$ theory which plays a special role in this theory. The action corresponding to this brane in the Einstein frame can be interpreted as a generalization of four dimensional $B I$ action coupled to a special background metric, dilaton, axion, etc. [5].

$$
\begin{aligned}
S_{D 3}= & -T_{D 3} \int d^{4} x \sqrt{-\operatorname{det}\left(g_{a b}+e^{-\phi / 2} B_{a b}\right)} \\
& +T_{D 3} \int\left[C^{(4)}+C^{(2)} B+\frac{1}{2} C^{(0)} B B\right],
\end{aligned}
$$

where all the bulk fields in the action are pulled back onto the world volume of D-brane.

It has been demonstrated that the $\mathrm{D}_{3}$-brane action (combined with type $I I B$ effective action) and the corresponding equation of motion and energy-momentum tensor are invariant under the $S L(2, R)$ symmetry of type $I I B$ theory $^{2}[3]$. In fact, this action satisfies the NGZ identity. As was pointed out in [6], the duality invariance of Hamiltonian and, thus, of the corresponding energy momentum tensor should imply the invariance of the $S$-matrix.

\footnotetext{
${ }^{2}$ This symmetry is not shared by the full type $I I B$ superstring theory. Indeed, it is broken by a variety of stringy and quantum effects to the infinite discrete subgroup $S L(2, Z)$.
} 
It is known that the $S$-matrix elements satisfy the Ward identity corresponding to the $S$-duality [7]. At the level of the equations of motion, $\mathrm{D}_{3}$-brane effective action is invariant under nonlinear $S$-duality transformation [3]. So, at higher orders, one does not expect the effective action to be consistent with the nonlinear $S$-duality.

It was pointed in [8] that the $B I$ action involving the onshell gauge field is invariant under the linear $S$-duality up to $F^{4}$ terms. From this point, the disk-level scattering amplitude of four gauge fields on the world volume of a single $\mathrm{D}_{3}$-brane, which appears as a contact term, should be invariant under the linear $S$-duality. The leading order $S$-matrix element of the six gauge fields has both contact terms coming from the $F^{6}$ terms in the $B I$ action and massless poles coming from the $F^{4}$ terms in the $B I$ action. So, as expected, no part of this amplitude is not invariant under the linear $S L(2, R)$ transformation separately; however, the combination of these two parts is invariant [9]. It has been discussed in [8] that by imposing the linear $S$-duality transformation on the $S$-dual invariant amplitude of four gauge fields, the pole part of the amplitude of six gauge fields could be constructed. Then, $F^{6}$-contact terms could be found by applying the linear $S$-duality again. Then using the linear $S$-duality, one may find the contact terms of $F^{8}$, and so on. This result confirms the $B I$ action with Abelian gauge fields as the effective action of a single D-brane.

So, in order to investigate the behavior of $S$-matrix elements under duality transformation, it is convenient to separate the $\alpha^{\prime}$ expansion of them into two parts: contact term and massless $n$-poles,

$$
\mathcal{A}=\mathcal{A}_{\text {contact }}+\sum_{n} A_{n-\text { poles }} .
$$

Neither $\mathcal{A}_{\text {contact }}$ nor $A_{n \text {-poles }}$ are invariant under the linear $S$-duality. In fact, the combination of these two parts, i.e., $\mathcal{A}$, must be invariant. On the other hand, the pole part of amplitude may transform to the contact part under the linear $S$-duality transformation, so the effective action, that is made of the contact part, satisfies the Ward identity corresponding to $S$-duality. ${ }^{3}$ In the case that the $S$-matrix elements have no massless poles, one can find the corresponding couplings from the consistency of them with the dualities [11].

Unlike the gauge field transformation that is carried by its field strength, all other transformations are done by field potential, so the pole part of the $S$-matrix does not transform to the contact part under the closed string $S L(2, R)$ transformation. It could be noted that if the $S$-matrix involves the antisymmetric NSNS (B-field) closed string,

\footnotetext{
${ }^{3}$ On the other hand, the $T$-duality transformation does not transform the pole terms to contact terms, so the contact terms always satisfy the Ward identity corresponding to $T$-duality [10].
}

one could find the amplitude in terms of $B$-field strength $H$ by combining the pole part in the amplitude which is produced by the gauge fields and some of the contact terms which are produced by the replacement of $F \rightarrow F+B$ [7].

The $S$-matrix elements of one $R R$ two-form field and three gauge fields that appear as a pole term and the $S$-matrix elements of one $B$ field and three gauge fields that contain a contact term as well as a pole term have been calculated in [9]. It has been shown that the combination of these two amplitudes could appear in terms of a $S L(2, R)$ invariant structure. In this paper we show that these amplitudes transform to each other under the linear $S$-duality transformation

$\mathcal{A}_{1-\text { pole }}\left(C^{2}, F^{3}\right) \rightarrow \mathcal{A}_{\text {contact }}\left(B, F^{3}\right)+\mathcal{A}_{1-\text { pole }}\left(B, F^{3}\right)$.

Inspired by the above observation that the $S$-matrix elements of one closed string and three open strings should satisfy the Ward identity corresponding to the $S$-duality, it could be proposed that the following expression for the massless n-pole amplitude of a $R R$ two-form.

$$
\begin{aligned}
\mathcal{A}_{n-\text { pole }} & \left(\left(C^{2}\right)^{n}, F^{4-n}\right) \\
\rightarrow & \mathcal{A}_{\text {contact }}\left(B^{n}, F^{4-n}\right)+\mathcal{A}_{1-\text { pole }}\left(B^{n}, F^{4-n}\right)+\cdots \\
& +\mathcal{A}_{n-\text { pole }}\left(B^{n}, F^{4-n}\right),
\end{aligned}
$$

where $n=0,1,2,3,4$. The contact part of the above amplitude could be exactly reproduced by the replacement of $F \rightarrow F+B$ in the $\mathrm{D}_{3}$-brane effective action.

It is convenient to consider the normalization factor $2 \pi \alpha^{\prime}$ in front of the gauge field in $D B I$ action. With this normalization, the $D B I$ action is at the leading order of $\alpha^{\prime}$. The $\alpha^{\prime}$ corrections to the Born-Infeld action have been studied in [12-17] in the $\sigma$-model approach.

The derivatives of gauge fields could appear in $\mathrm{D}_{3}$-brane effective action. The study of the behavior of four point function under the linear $S$-duality could be extended to four point function with derivative corrections [18]. It has been shown in [19] that the electromagnetic self-duality holds for such functions with derivative corrections at the order of $\alpha^{\prime 2}$. So, one might expect that the $S L(2, R) S$-dual invariant structure could appear for each order of $\alpha^{\prime}$ independently. On other words, we have to answer the following question: Should $S$-duality be a symmetry of full $\mathrm{D}_{3}$-brane action with all derivative corrections? There is no exact reasoning for this, but there is still an alternative (albeit somewhat exploratory) logic suggesting that $\mathrm{D}_{3}$-brane action (but not actions for other D-branes!) should be $S$-duality covariant ${ }^{4}$ [3] (see also [20-22]).

By calculating the scattering amplitude of four open strings in $I I B$ superstring theory, it has been found that the

\footnotetext{
${ }^{4}$ We would like to thank A. A. Tseytlin for discussions on this point.
} 
leading order of $\mathrm{D}_{3}$-brane effective action in superstring theory contains four gauge fields and four derivatives $[15,19,23]$ :

$$
\begin{aligned}
L_{(\partial F)^{4}}= & e^{-2 \phi}\left(\frac{1}{4} \partial_{c} F^{e f} \partial^{c} F^{a b} \partial_{d} F_{e f} \partial^{d} F_{a b}\right. \\
& -\frac{1}{2} \partial_{c} F_{d f} \partial^{c} F^{a b} \partial_{e} F_{b}{ }^{f} \partial^{e} F_{a}{ }^{d} \\
& -\partial_{c} F_{a}{ }^{d} \partial^{c} F^{a b} \partial_{f} F_{d e} \partial^{f} F_{b}{ }^{e} \\
& \left.+\frac{1}{8} \partial_{c} F_{a b} \partial^{c} F^{a b} \partial_{f} F_{d e} \partial^{f} F^{d e}\right) .
\end{aligned}
$$

This action satisfies the NGZ identity, and is consistent with electromagnetic self-duality [19]. We will find the form of the above action that is manifestly $S$-dual. In fact, we will find the above action in terms of a nonlinear $S$-dual structure. From the nonlinear expansion of the $S L(2, R)$ invariant structures, we find this effective action up to the level of $N$ gauge fields and four derivatives.

The outline of the paper is as follows: We begin in Sec. II by studying the $S$-duality transformations of bosonic fields and find some $S L(2, R)$ invariant structures involving these fields. In Sec. III, we show that the compatibility of the $S$-matrix elements of one gauge field, two scalar fields and one $R R$ field $C^{(2)}$ with the $S$-duality generates the $S$-matrix elements of one gauge field, two scalar fields and one $N S N S B$-field. We find a $S$-dual invariant form of these amplitudes and then predict the axion amplitude. In Sec. IV, we calculate the $S$-matrix elements of three gauge fields and one NSNS $B$-field by applying the $S$-duality transformation on the $S$-matrix elements of three gauge fields and one $R R$ field $C^{(2)}$. In Sec. V, using $S L(2, R)$ invariant structures found in Sec. II, we write the $\mathrm{D}_{3}$-brane effective action involving four gauge fields and four derivatives in $S$-dual form. This expression could be extended to include more gauge fields.

\section{NONLINEAR $S L(2, R)$ STRUCTURE}

The axion $C_{0}$ combines with the dilaton $\phi_{0}$ to give the complex scalar field $\tau=C_{0}+i e^{-\phi_{0}}$. Now consider the matrix $\mathcal{M}$ :

$$
\mathcal{M}=e^{\phi_{0}}\left(\begin{array}{cc}
|\tau|^{2} & C_{0} \\
C_{0} & 1
\end{array}\right)
$$

The nonlinear $S L(2, R)$ transformation of the complex scalar field and the above matrix are given by [24]

$$
\tau \rightarrow \frac{p \tau+q}{r \tau+s}, \quad \mathcal{M} \rightarrow \Lambda \mathcal{M} \Lambda^{T} ; \quad \Lambda=\left(\begin{array}{cc}
p & q \\
r & s
\end{array}\right) \in S L(2, R) .
$$

For the gauge field we have the following transformation:

$$
F_{a b} \rightarrow s F_{a b}+r \tilde{G}_{a b}, \quad G_{a b} \rightarrow p G_{a b}-q \tilde{F}_{a b},
$$

where $F_{a b}=-\frac{1}{2} \epsilon_{a b c d} \tilde{F}^{c d}$, the antisymmetric tensor $G_{a b}$ is given by $-2 \partial \mathcal{L}(F) / \partial F^{a b}$, and $\mathcal{L}$ is the Lagrangian. By considering a gauge field doublet $\mathcal{F}_{a b}$ containing $F_{a b}$ and $G_{a b}$ and also a background field doublet $\mathcal{B}_{a b}$ containing the two forms $B^{(2)}$ and $C^{(2)}$ and using $(\tilde{F})=-F$, one can write the transformation of these field doublets as

$$
\mathcal{F}_{a b} \rightarrow\left(\Lambda^{-1}\right)^{T} \mathcal{F}_{a b} ; \quad \mathcal{B}_{a b} \rightarrow\left(\Lambda^{-1}\right)^{T} \mathcal{B}_{a b} .
$$

Using the above transformations, one can find that the structures $\mathcal{F}^{T} \mathcal{M F}$ and $\mathcal{B}^{T} \mathcal{M B}$ are $S L(2, R)$ invariant structures.

Consider the Lagrangian in terms of two possible Lorentz invariants: $t=1 / 4 F^{a b} F_{a b}$ and $z=1 / 4 F^{a b} \tilde{F}_{a b}$. At the presence of axion and dilaton couplings, it could be useful to express the Lagrangian in the following form which the contribution of axion coupling is separated:

$$
\mathcal{L}=\mathcal{L}^{\prime}+C_{0} z
$$

The antisymmetric tensor $G_{a b}$ then separates as $G_{a b}=$ $G_{a b}^{\prime}-C_{0} \tilde{F}_{a b}$, where $G_{a b}^{\prime}$ could be an arbitrary nonlinear function of $F$, which is $G_{a b}^{\prime}=-2 \frac{\partial \mathcal{L}^{\prime}}{\partial F^{a b}}$.

By this consideration one gets the $S L(2, R)$ invariant structure in the following form:

$$
\left(\mathcal{F}^{T}\right)_{a}{ }^{c} \mathcal{M}_{0} \mathcal{F}_{b c}=e^{-\phi_{0}} \tilde{F}_{a}{ }^{c} \tilde{F}_{b c}+e^{\phi_{0}} G_{a}^{\prime c} G_{b c}^{\prime},
$$

where the matrix $\mathcal{M}_{0}$ is the matrix $\mathcal{M}$ in which the dilaton and the R-R scalar are constant $\left(\phi_{0}, C_{0}\right)$. It is easy to check that the above structure is invariant under the linear transformations $G^{\prime} \rightarrow \tilde{F}, \tilde{F} \rightarrow-G^{\prime}$, and $e^{-\phi_{0}} \rightarrow e^{\phi_{0}}$.

When the dilaton and the R-R scalar are constant, one finds the following $S$-dual multiplet [25]:

$$
\left(\tilde{\mathcal{F}}^{T}\right)_{a}{ }^{c} \mathcal{M}_{0} \mathcal{B}_{b c}=e^{-\phi_{0}} F_{a}{ }^{c} B_{b c}-\tilde{F}_{a}{ }^{c} C_{b c}^{(2)}-C_{0} \tilde{F}_{a}{ }^{c} B_{b c} .
$$

At the presence of two gauge fields with two derivatives, one can find the following $S L(2, R)$ invariant structure:

$$
\partial \mathcal{F}^{T} \mathcal{M} \partial \mathcal{F}=e^{-\phi_{0}} \partial \tilde{F} \partial \tilde{F}+e^{\phi_{0}} \partial G^{\prime} \partial G^{\prime}
$$

By considering the nonlinear expansion of $G_{a b}^{\prime}$ (in any self dual theory) as $G_{a b}^{\prime}=e^{-\phi_{0}} F+e^{-2 \phi_{0}} F^{3}+\cdots+e^{-N \phi_{0}} F^{2 N-1}$, the nonlinear $S L(2, R)$ invariant structure corresponding to $2 N$ gauge fields and two derivatives could be found.

$\partial \mathcal{F}^{T} \mathcal{M} \partial \mathcal{F} \sim \partial F \partial F+F F \partial F \partial F+F F F F \partial F \partial F+\cdots$. 


\section{III. $S$-DUAL AMPLITUDE OF ONE GAUGE FIELD, TWO SCALAR FIELDS, AND ONE TWO-FORM}

The $S$-matrix elements of one gauge field, two scalar fields, and one two-form vertex operator could be calculated from $\mathrm{D}_{3}$-brane effective action. At first, we are going to find the corresponding amplitude in which the two-form is an antisymmetric $R R$ field $C^{(2)}$. The amplitude is given by the following Feynman rule:

$$
\begin{aligned}
& \mathcal{A}\left(C_{1}^{(2)}, F_{2}, \Phi_{3}, \Phi_{4}\right) \\
& \quad=V^{a}\left(C_{1}^{(2)}, A\right) G_{a b}(A) V^{b}\left(A, F_{2}, \Phi_{3}, \Phi_{4}\right),
\end{aligned}
$$

where $F_{2}$ is the polarization of the gauge boson, $\Phi_{3}, \Phi_{4}$ are the scalar fields, and $A$ is the off-shell gauge field propagating between the two vertices. The gauge boson propagator and the vertices can be read from $\mathrm{D}_{3}$-brane effective action

$$
\begin{aligned}
G_{a b}(A) & \sim-\frac{e^{\phi_{0}} \eta_{a b}}{k \cdot k} \\
V^{a}\left(C_{1}^{(2)}, A\right) & \sim-k \cdot\left(\tilde{C}_{1}{ }^{(2)}\right)^{a} \\
V^{b}\left(A, F_{2}, \Phi_{3}, \Phi_{4}\right) & \sim e^{-\phi_{0}} \Phi_{3 i} \Phi_{4}{ }^{i}\left[k \cdot k_{3} k_{4} \cdot F_{2}^{a}+k \cdot F_{2} \cdot k_{4} k_{3}^{a}\right. \\
& \left.-\frac{1}{2} k_{3} \cdot k_{4} k \cdot F_{2}^{a}\right]+(3 \leftrightarrow 4),
\end{aligned}
$$

where $\left(\tilde{C}^{(2)}\right)_{a b}=\frac{1}{2} \epsilon_{a b c d}\left(C^{(2)}\right)^{c d}$. From the conservation of momentum along the brane we have $k=-k_{1}=k_{2}+k_{3}+k_{4}$. Considering the above vertices and propagator, one finds that the $S$-matrix elements have no contribution to the contact amplitude, and the amplitude (14) then appears as a pole amplitude:

$$
\begin{aligned}
\mathcal{A}\left(C_{1}^{(2)}, F_{2}, \Phi_{3}, \Phi_{4}\right)= & \frac{\Phi_{3 i} \Phi_{4}^{i}}{k_{1} \cdot k_{1}}\left[k_{1} \cdot \tilde{C}_{1}^{(2)} \cdot k_{4} k_{1} \cdot F_{2} \cdot k_{3}\right. \\
& -k_{1} \cdot k_{3} k_{1} \cdot \tilde{C}_{1}^{(2)} \cdot F_{2} \cdot k_{4} \\
& \left.+\frac{1}{2} k_{3} \cdot k_{4} k_{1} \cdot \tilde{C}_{1}^{(2)} \cdot F_{2} \cdot k_{1}\right] \\
& +(3 \leftrightarrow 4) .
\end{aligned}
$$

The amplitude does not satisfy the Ward identity corresponding to the $S$-duality transformations; however, it is invariant under the Ward identity corresponding to the Abelian gauge symmetry and to the $R R$ gauge symmetry.

The amplitude corresponding to antisymmetric $B$-field has a massless pole as well as a contact term. The contact part of the $B$-field amplitude can be found using the Feynman rule and considering the relevant higher derivative correction of D-brane action.

$$
\begin{aligned}
\mathcal{A}_{\text {contact }}\left(B_{1}, F_{2}, \Phi_{3}, \Phi_{4}\right)= & e^{-\phi_{0}} \Phi_{3 i} \Phi_{4}^{i}\left[\frac{1}{4} k_{3} \cdot k_{4} \operatorname{Tr}\left(B_{1} \cdot F_{2}\right)\right. \\
& \left.-k_{3} \cdot B_{1} \cdot F_{2} \cdot k_{4}\right]+(3 \leftrightarrow 4) .
\end{aligned}
$$

Considering the propagator and vertex $V^{b}\left(A, F_{2}, \Phi_{3}, \Phi_{4}\right)$ in (16) and using the fourth order in the expansion of D-brane action to find the vertex operator $V^{a}\left(B_{1}, A\right)=e^{-\phi_{0}} k_{1} \cdot B_{1}^{a}$, one finds the pole part of $B$-field amplitude as following:

$$
\begin{aligned}
\mathcal{A}_{\text {pole }}\left(B_{1}, F_{2}, \Phi_{3}, \Phi_{4},\right)= & V^{a}\left(B_{1}, A\right) G_{a b}(A) V^{b}\left(A, F_{2}, \Phi_{3}, \Phi_{4}\right) \\
= & -\frac{e^{-\Phi} \Phi_{3 i} \Phi_{4}^{i}}{k_{1} \cdot k_{1}}\left[k_{1} \cdot B_{1} \cdot k_{4} k_{1} \cdot F_{2} \cdot k_{3}\right. \\
& -k_{1} \cdot k_{3} k_{1} \cdot B_{1} \cdot F_{2} \cdot k_{4} \\
& \left.+\frac{1}{2} k_{3} \cdot k_{4} k_{1} \cdot B_{1} \cdot F_{2} \cdot k_{1}\right] \\
& +(3 \leftrightarrow 4),
\end{aligned}
$$

where we use the previously mentioned conservation of momentum.

Now we are going to determine the transformation behavior of the amplitude (16) under the $S$-duality transformation when the axion background does not take into account $C_{0}=0$. There are two different structures in amplitude (16) that transform under the $S$-duality as follows:

$$
\begin{aligned}
k_{N} \cdot \tilde{C}_{1}^{(2)} \cdot F_{2} \cdot k_{M} \rightarrow e^{-\phi} k_{N} \cdot \tilde{B}_{1} \cdot \tilde{F}_{2} \cdot k_{M}= & e^{-\phi}\left(k_{N} \cdot F_{2} \cdot B_{1} \cdot k_{M}-\frac{1}{2} k_{N} \cdot k_{M} \operatorname{Tr}\left(B_{1} \cdot F_{2}\right)\right) \\
k_{N} \cdot \tilde{C}_{1}^{(2)} \cdot k_{M} k_{P} \cdot F_{2} \cdot k_{Q} \rightarrow e^{-\phi} k_{N} \cdot \tilde{B}_{1} \cdot k_{M} k_{P} \cdot \tilde{F}_{2} \cdot k_{Q}= & e^{-\phi}\left(-\frac{1}{2} k_{N} \cdot k_{Q} k_{P} \cdot k_{M} \operatorname{Tr}\left(B_{1} \cdot F_{2}\right)+\frac{1}{2} k_{N} \cdot k_{P} k_{Q} \cdot k_{M} \operatorname{Tr}\left(B_{1} \cdot F_{2}\right)\right. \\
& -k_{N} \cdot F_{2} \cdot k_{M} k_{P} \cdot B_{1} \cdot k_{Q}-k_{Q} \cdot k_{M} k_{N} \cdot F_{2} \cdot B_{1} \cdot k_{P} \\
& +k_{P} \cdot k_{M} k_{N} \cdot F_{2} \cdot B_{1} \cdot k_{Q}+k_{N} \cdot k_{Q} k_{M} \cdot F_{2} \cdot B_{1} \cdot k_{P} \\
& \left.-k_{N} \cdot k_{P} k_{M} \cdot F_{2} \cdot B_{1} \cdot k_{Q}\right)
\end{aligned}
$$


where we use the following identity:

$$
\epsilon^{a b c d} \epsilon^{e f g h}=-\left|\begin{array}{llll}
\eta^{a e} & \eta^{a f} & \eta^{a g} & \eta^{a h} \\
\eta^{b e} & \eta^{b f} & \eta^{b g} & \eta^{b h} \\
\eta^{c e} & \eta^{c f} & \eta^{c g} & \eta^{c h} \\
\eta^{d e} & \eta^{d f} & \eta^{d g} & \eta^{d h}
\end{array}\right| .
$$

Applying the above relations in the amplitude (16), one obtains the amplitudes (17) and (18). In fact the amplitude of one $R R$, two scalars, and one gauge field transform to the amplitude of one NSNS B-field, two scalars, and one gauge field under the $S$-duality.

$$
\begin{aligned}
\mathcal{A}\left(C_{1}^{(2)}, F_{2}, \Phi_{3}, \Phi_{4}\right) \rightarrow & \mathcal{A}_{\text {contact }}\left(B_{1}, F_{2}, \Phi_{3}, \Phi_{4}\right) \\
& +\mathcal{A}_{\text {pole }}\left(B_{1}, F_{2}, \Phi_{3}, \Phi_{4}\right) .
\end{aligned}
$$

Therefore, to get the NSNS coupling to brane, one can apply the $S$-duality on the pole amplitude containing the $R R$ state. This is consistent with the statement that the NSNS coupling is found by replacement of $F \rightarrow F+B$ in the D-brane effective action that has been expressed in the literature.

At the presence of the axion field, it is expected that one can find the combination of the amplitudes (16), (17), and (18) in terms of $S L(2, R)$ invariant structures. To do this, we consider all contractions of the $S L(2, R)$ invariant structure $\tilde{\mathcal{F}}^{T} \mathcal{M B}$ with four, two, and zero momenta, as follows:

$$
\begin{aligned}
\left(\tilde{\mathcal{F}}_{N}^{T}\right)^{a b} \mathcal{M B}_{M}^{c d} k_{1 a} k_{2 b} k_{3 c} k_{4 d}= & e^{-\phi} k_{1} \cdot F_{N} \cdot k_{2} k_{4} \cdot B_{M} \cdot k_{3}+C_{0} e^{-\phi} k_{1} \cdot F_{N} \cdot k_{2} k_{4} \cdot \tilde{B}_{M} \cdot k_{3}-\frac{1}{2} k_{4} \cdot k_{2} k_{1} \cdot k_{3} \operatorname{Tr}\left(F_{N} \cdot \tilde{C}_{M}\right) \\
& +\frac{1}{2} k_{1} \cdot k_{4} k_{2} \cdot k_{3} \operatorname{Tr}\left(F_{N} \cdot \tilde{C}_{M}\right)+k_{3} \cdot F_{N} \cdot k_{4} k_{1} \cdot \tilde{C}_{M} \cdot k_{2}-k_{2} \cdot k_{3} k_{4} \cdot F_{N} \cdot \tilde{C}_{M} \cdot k_{1} \\
& +k_{1} \cdot k_{3} k_{4} \cdot F_{N} \cdot \tilde{C}_{M} \cdot k_{2}+k_{4} \cdot k_{2} k_{3} \cdot F_{N} \cdot \tilde{C}_{M} \cdot k_{1}-k_{1} \cdot k_{4} k_{3} \cdot F_{N} \cdot \tilde{C}_{M} \cdot k_{2}, \\
k_{3} \cdot \tilde{\mathcal{F}}_{N}^{T} \mathcal{M} \mathcal{B}_{M} \cdot k_{4}= & -e^{-\phi} k_{3} \cdot F_{N} \cdot B_{M} \cdot k_{4}-C_{0} e^{-\phi} k_{3} \cdot F_{N} \cdot \tilde{B}_{M} \cdot k_{4} \\
& +\frac{1}{2} k_{3} \cdot k_{4} \operatorname{Tr}\left(F_{N} \cdot \tilde{C}_{M}\right)-k_{3} \cdot \tilde{C}_{M} \cdot F_{N} \cdot k_{4}, \\
\operatorname{Tr}\left(\tilde{\mathcal{F}}_{N}^{T} \mathcal{M B}_{M}\right)= & -e^{-\phi} \operatorname{Tr}\left(F_{N} \cdot B_{M}\right)-C_{0} e^{-\phi} \operatorname{Tr}\left(F_{N} \cdot B_{M}\right)+\operatorname{Tr}\left(F_{N} \cdot \tilde{C}_{M}\right) .
\end{aligned}
$$

Considering the above relations, one can find the following combination of the above $\operatorname{SL}(2, R)$ invariant structures contains the amplitude (16) as well as the amplitude (17) + (18).

$$
\begin{aligned}
\tilde{\mathcal{A}}= & -\frac{\Phi_{3 i} \Phi_{4}^{i}}{k_{1} \cdot k_{1}}\left[\frac{1}{2} k_{3} \cdot k_{4} k_{1} \cdot \tilde{\mathcal{F}}_{2}^{T} \mathcal{M B}_{1} \cdot k_{1}\right. \\
& \left.-k_{1} \cdot k_{4} k_{3} \cdot \tilde{\mathcal{F}}_{2}^{T} \mathcal{M} \mathcal{B}_{1} \cdot k_{1}-k_{1} \cdot \tilde{\mathcal{F}}_{2}^{T} \cdot k_{3} \mathcal{M} k_{4} \cdot \mathcal{B}_{1} \cdot k_{1}\right] \\
& +\Phi_{3 i} \Phi_{4}^{i}\left[k_{3} \cdot \tilde{\mathcal{F}}_{2}^{T} \mathcal{M} \mathcal{B}_{1} \cdot k_{4}-\frac{1}{4} k_{3} \cdot k_{4} \operatorname{Tr}\left(\tilde{\mathcal{F}}_{2}^{T} \mathcal{M} \mathcal{B}_{1}\right)\right] \\
& +(3 \leftrightarrow 4) .
\end{aligned}
$$

It is clear that this $S L(2, R)$ invariant amplitude [that is manifestly invariant under the $S L(2, R)$ transformation] also contains the amplitude $\mathcal{A}\left(C_{0} ; B_{1}, F_{2}, \Phi_{3}, \Phi_{4}\right)$. In fact, to make a $S L(2, R)$ invariant amplitude from amplitudes (16) and (17) + (18), the amplitude corresponding to the axion field should be take into account. On the other hand, we can confirm the above $S L(2, R)$ invariant amplitude by deriving the axion amplitude from the explicit calculation and the Feynman rule that results in the following pole amplitude:

$$
\begin{aligned}
& \mathcal{A}\left(C_{0} ; B_{1}, F_{2}, \Phi_{3}, \Phi_{4}\right) \\
&=-\frac{C_{0} e^{-\phi} \Phi_{3 i} \Phi_{4}^{i}}{k_{1} \cdot k_{1}}\left[k_{1} \cdot \tilde{B}_{1} \cdot k_{4} k_{1} \cdot F_{2} \cdot k_{3}\right. \\
&-k_{1} \cdot k_{3} k_{1} \cdot \tilde{B}_{1} \cdot F_{2} \cdot k_{4} \\
&\left.+\frac{1}{2} k_{3} \cdot k_{4} k_{1} \cdot \tilde{B}_{1} \cdot F_{2} \cdot k_{1}\right]+(3 \leftrightarrow 4) .
\end{aligned}
$$

This is exactly equal to the axion amplitude that lies in (22).

\section{IV. $S$-DUAL AMPLITUDE OF THREE GAUGE FIELDS AND ONE TWO-FORM}

It has been shown that the amplitude of three gauge fields and one two-form in which the two-form is $R R$ field $C^{(2)}$ or $N S N S B$-field could appear in terms of $S L(2, R)$ invariant structures in the presence of the background dilaton and R-R scalar fields [9]. We are going to show that these two amplitudes transform to each other under the $S$-duality transformation, which the axion field does not take into account. We start with the amplitude of three gauge fields and one $R R$ field $C^{(2)}$ that is found by explicit calculation $[9,26]$ : 


$$
\begin{aligned}
\mathcal{A}\left(C_{1}{ }^{(2)}, F_{2}, F_{3}, F_{4}\right)= & \frac{1}{4} e^{-\phi_{0}} \frac{k_{1} \cdot F_{2} \cdot \tilde{C}_{1}^{(2)} \cdot k_{1}}{k_{1} \cdot k_{1}} \operatorname{Tr}\left(F_{3} \cdot F_{4}\right) \\
& -e^{-\phi_{0}} \frac{k_{1} \cdot F_{2} \cdot F_{3} \cdot F_{4} \cdot \tilde{C}_{1}^{(2)} \cdot k_{1}}{k_{1} \cdot k_{1}} \\
& +P(2,3,4),
\end{aligned}
$$

where $P(2,3,4)$ stands for the other permutations of $2,3,4$. This amplitude contains two independent structures. We apply $S$-duality transformation to these two structures separately.

$$
\begin{aligned}
& e^{-\phi_{0}} k_{1} \cdot F_{2} \cdot F_{3} \cdot F_{4} \cdot \tilde{C}^{(2)} \cdot k_{1} \\
& \quad \stackrel{s}{\rightarrow} \frac{e^{-2 \phi_{0}}}{16} k_{1 a} \epsilon^{a b k l} \epsilon_{b c n m} \epsilon^{c d g h} \epsilon_{d e i j} F_{2 k l} F_{3}{ }^{n m} F_{4 g h} B^{i j} k_{1}{ }^{e}=I_{234}, \\
& e^{-\phi_{0}} k_{1} \cdot F_{2} \cdot \tilde{C}^{(2)} \cdot k_{1} \operatorname{Tr}\left(F_{3} \cdot F_{4}\right) \\
& \quad \stackrel{S}{\rightarrow} \frac{e^{-2 \phi_{0}}}{16} k_{1 a} \epsilon^{a b k l} \epsilon_{b c n m} \epsilon^{d e g h} \epsilon_{d e i j} F_{2 k l} F_{3 g h} F_{4}{ }^{i j} B^{n m} k_{1}{ }^{e}=J_{234} .
\end{aligned}
$$

Because of the invariance of the trace term in the original amplitude under the gauge fields permutations, one can easily conclude $J_{234}=J_{243}, J_{423}=J_{432}$, and $J_{324}=J_{342}$ in the dual amplitude. Therefore, the gauge field permutations can produce six different terms of $I$-type and three different terms of $J$-type that contribute in the dual amplitude.

The calculation in this case is more complicated than the case in the previous section due to the presence of four Levi-Civita tensors. To rewrite these terms in terms of various contractions of gauge fields, one has to use the identity (19). Here, one encounters with different choices. In fact, there are three ways to pairs the Levi-Civita tensors: $\mathcal{E}=\left(\epsilon^{a b k l} \epsilon_{b c n m}\right)\left(\epsilon^{\text {degh }} \epsilon_{d e i j}\right), \quad \mathcal{E}^{\prime}=\left(\epsilon^{a b k l} \epsilon_{d e i j}\right)\left(\epsilon^{c d g h} \epsilon_{b c n m}\right)$, and $\mathcal{E}^{\prime \prime}=\left(\epsilon^{a b k l} \epsilon^{c d g h}\right)\left(\epsilon_{b c n m} \epsilon_{d e i j}\right)$. Using the standard identity (19) for the above first two pairings in I-type terms, we have:

$$
\begin{aligned}
I_{N M P}(\mathcal{E})= & e^{-2 \phi_{0}}\left[-k_{1} \cdot F_{N} \cdot B \cdot F_{P} \cdot F_{M} \cdot k_{1}-k_{1} \cdot F_{N} \cdot F_{M} \cdot F_{P} \cdot B \cdot k_{1}-k_{1} \cdot F_{3} \cdot F_{M} \cdot F_{N} \cdot B \cdot k_{1}\right. \\
& +\frac{1}{2} k_{1} \cdot F_{P} \cdot F_{M} \cdot k_{1} \operatorname{Tr}\left(F_{N} \cdot B\right)+\frac{1}{2} k_{1} \cdot F_{N} \cdot B \cdot k_{1} \operatorname{Tr}\left(F_{P} \cdot F_{M}\right) \\
& \left.-\frac{1}{4} k_{1} \cdot k_{1} \operatorname{Tr}\left(F_{N} \cdot B\right) \operatorname{Tr}\left(F_{P} \cdot F_{M}\right)+k_{1} \cdot k_{1} \operatorname{Tr}\left(F_{N} \cdot F_{M} \cdot F_{P} \cdot B\right)\right], \\
I_{N M P}\left(\mathcal{E}^{\prime}\right)= & e^{-2 \phi_{0}}\left[k_{1} \cdot F_{P} \cdot B \cdot F_{N} \cdot F_{M} \cdot k_{1}-\frac{1}{2} k_{1} \cdot F_{P} \cdot B \cdot k_{1} \operatorname{Tr}\left(F_{N} \cdot F_{M}\right)\right. \\
& \left.-\frac{1}{2} k_{1} \cdot F_{N} \cdot F_{M} \cdot k_{1} \operatorname{Tr}\left(F_{P} \cdot B\right)+\frac{1}{4} k_{1} \cdot k_{1} \operatorname{Tr}\left(F_{N} \cdot F_{M}\right) \operatorname{Tr}\left(F_{P} \cdot B\right)\right],
\end{aligned}
$$

and for the first pairing in the $J$-type term, we have

$$
J_{N M P}(\mathcal{E})=e^{-2 \phi_{0}}\left[-k_{1} \cdot F_{N} \cdot B \cdot k_{1} \operatorname{Tr}\left(F_{M} \cdot F_{P}\right)+\frac{1}{2} k_{1} \cdot k_{1} \operatorname{Tr}\left(F_{N} \cdot B\right) \operatorname{Tr}\left(F_{M} \cdot F_{P}\right)\right],
$$

where $N, M, P=2,3,4$ and $N \neq M \neq P$.

Our calculations show that one can get to the amplitude of $\mathcal{A}\left(B_{1}{ }^{(2)}, F_{2}, F_{3}, F_{4}\right)$ only by selecting the specific pairings of Levi-Civita tensors as follows:

$\mathcal{A}\left(C_{1}{ }^{(2)}, F_{2}, F_{3}, F_{4}\right) \stackrel{S}{\rightarrow} \frac{I_{234}\left(\mathcal{E}^{\prime}\right)+I_{324}\left(\mathcal{E}^{\prime}\right)+I_{423}\left(\mathcal{E}^{\prime}\right)+I_{243}(\mathcal{E})+I_{342}(\mathcal{E})+I_{432}(\mathcal{E})}{k_{1} \cdot k_{1}}-\frac{1}{2} \frac{J_{234}(\mathcal{E})+J_{324}(\mathcal{E})+J_{423}(\mathcal{E})}{k_{1} \cdot k_{1}}$.

Replacing (25) and (26) in the above relation and using the conservation of momentum, one finds the following expression, after a straightforward calculation:

$$
\begin{aligned}
& \frac{1}{2} e^{-2 \phi_{0}}\left(\operatorname{Tr}\left(B_{1} \cdot F_{2} \cdot F_{3} \cdot F_{4}\right)-\frac{1}{4} \operatorname{Tr}\left(B_{1} \cdot F_{2}\right) \operatorname{Tr}\left(F_{3} \cdot F_{4}\right)\right)-\frac{1}{4} e^{-2 \phi_{0}} \frac{k_{1} \cdot F_{2} \cdot B_{1} \cdot k_{1}}{k_{1} \cdot k_{1}} \operatorname{Tr}\left(F_{3} \cdot F_{4}\right) \\
& \quad+e^{-2 \phi_{0}} \frac{k_{1} \cdot F_{2} \cdot F_{3} \cdot F_{4} \cdot B_{1} \cdot k_{1}}{k_{1} \cdot k_{1}}+P(2,3,4) .
\end{aligned}
$$

As we have mentioned in (3), this is exactly equal to the amplitude of one $B$-field and three gauge fields containing the pole term as well as the contact term that was calculated in [9]. 


\section{V. $S$-DUAL EFFECTIVE ACTION WITH FOUR DERIVATIVE CORRECTIONS}

It has been shown that the one-loop correction to the $\mathrm{D}_{3^{-}}$ brane Abelian Born-Infeld action (4) contains four gauge fields with four derivatives proportional to $\left(s^{2}+t^{2}+u^{2}\right) F^{4}$, where $s, t$, and $u$ are the Mandelstam variables [23]. This result has been found from supersymmetry fixing [27] and string Disk-level scattering amplitude calculation [28]. The $\mathrm{D}_{3}$-brane effective action at the order of $(\partial F)^{4}$ is invariant under the $S L(2, R)$ duality. From the Lagrangian viewpoint, this action saturates the NGZ identity [19].
We are going to find this action in terms of some $S L(2, R)$ invariant structures. To do this, one should consider all contractions of two nonlinear structures, $W^{a b c d e f g} W_{\text {ghnmpq }}$, any of which contains two gauge field derivative terms as follows:

$$
W^{\text {abcdef }}=\partial^{a}\left(\mathcal{F}^{T}\right)^{b c} \mathcal{M} \partial^{d} \mathcal{F}^{e f}
$$

Using "xAct" [29], one finds the following 33 different $W W$ contractions:

$$
\begin{aligned}
& w_{1} W_{c}{ }^{e f c a b} W_{d e f}{ }_{a b}^{d}+w_{2} W_{a}^{a b}{ }_{c}^{e f} W_{d e f}{ }^{d}{ }_{b}^{c}+w_{3} W_{b e f}{ }^{c a b} W_{d c}{ }^{f e}{ }_{a}{ }^{d}+w_{4} W_{b c}{ }^{f c a b} W_{d e f}{ }^{e}{ }_{a}^{d}+w_{5} W_{c d f}{ }^{c a b} W_{e b}{ }^{f e}{ }_{a}^{d} \\
& +w_{6} W_{a}{ }^{a b}{ }_{c b}{ }^{c} W_{d}{ }^{d e}{ }_{f e}^{f}+w_{7} W_{a}{ }^{a b}{ }_{c d}{ }^{e} W^{d}{ }_{b}{ }^{c} f e^{f}+w_{8} W_{a}{ }^{a b}{ }_{d c}{ }^{e} W^{d}{ }_{b}{ }^{c} f e^{f}+w_{9} W_{a}{ }^{a b}{ }_{b}{ }^{c d} W^{e}{ }_{c d f e} e^{f}+w_{10} W_{a}{ }^{a b d}{ }_{b}{ }^{c} W^{e}{ }_{c d f e}{ }^{f} \\
& +w_{11} W^{c a b e}{ }_{a}^{d} W_{f c e}{ }_{b d}+w_{12} W_{c d f}{ }^{c a b} W^{e}{ }_{a}^{d f}{ }_{b e}+w_{13} W^{c a b}{ }_{d c f} W_{a}^{e}{ }_{a}^{d f}{ }_{b e}+w_{14} W^{c a b e}{ }_{a}^{d} W_{f c d}{ }_{b e}+w_{15} W^{c a b}{ }_{d e f} W^{d}{ }_{a c}^{f}{ }_{b}^{e} \\
& +w_{16} W_{c a}{ }^{d c a b} W_{e d f}{ }_{b}{ }^{e}+w_{17} W^{c a b d}{ }_{a c} W_{e d f}{ }_{b}{ }^{e}+w_{18} W_{c a}{ }^{d c a b} W_{f d e}{ }_{b}{ }^{e}+w_{19} W^{c a b d}{ }_{a c} W_{f d e}{ }_{b}{ }^{e}+w_{20} W_{a}{ }^{a b}{ }_{b}{ }^{c d} W_{\text {def }}{ }^{e}{ }_{c}^{e} \\
& +w_{21} W^{c a b}{ }_{d e f} W^{d}{ }_{a b}{ }^{f}{ }_{c}^{e}+w_{22} W_{a}^{a b}{ }_{d e f} W_{b}^{d}{ }_{b}{ }^{c f}{ }_{c}^{e}+w_{23} W^{c a b d}{ }_{a b} W_{e d f}{ }_{c}^{e}+w_{24} W_{a}{ }^{a b d}{ }_{b}{ }^{c} W_{e d f}{ }_{c}^{e}+w_{25} W^{c a b d}{ }_{a b} W_{f d e}{ }_{c}^{e} \\
& +w_{26} W_{a}{ }^{a b d}{ }_{b}{ }^{c} W_{f d e}{ }_{c}{ }_{c}^{e}+w_{27} W_{b e f}{ }^{c a b} W^{d}{ }_{a c}^{f}{ }_{d}^{e}+w_{28} W_{a}^{a b}{ }_{c e f} W^{d}{ }_{b}{ }^{c f}{ }_{d}^{e}+w_{29} W_{a}{ }^{a b}{ }_{c b}{ }^{c} W_{e d f} f d e+w_{30} W_{b a c}{ }^{c a b} W_{e d f}{ }^{f d e} \\
& +w_{31} W_{c a b}{ }^{c a b} W_{e d f}{ }^{f d e}+w_{32} W_{a}{ }^{a b}{ }_{c b}{ }^{c} W_{f d e}{ }^{f d e}+w_{33} W_{c a b}{ }^{c a b} W_{f d e}{ }^{f d e} \text {, }
\end{aligned}
$$

where $w_{i}$ are some unknown coefficients that would be fixed by imposing some physical identity.

Using the $S L(2, R)$ invariant structure (12) at the level of four gauge fields and four derivatives, one finds that the $W W$ structures appear in terms of the gauge field contents $(\partial F)^{4},(\partial F)^{2}(\partial \tilde{F})^{2}$, and $(\partial \tilde{F})^{4}$. In order to make the action (4) in terms of the above $W W$ structures, we should find the contribution of dual gauge field $\tilde{F}$ in the last two field contents in terms of gauge field $F$ by using the identity (19). For the third field content, we encounter with four Levi-Civita tensors and with different choices to pair these tensors as was mentioned in the previous section: $\mathcal{E}, \mathcal{E}^{\prime}$, and $\mathcal{E}^{\prime \prime}$. Any of these pairings of Levi-Civita tensors led to different results in which the gauge fields appear in different kinds of contraction terms $(\partial F)^{4}$, but one can show that these results are equal when the identities presenting in the Appendix have been taken into account.

After finding the above $W W$ structures in terms of gauge field contraction terms $(\partial F)^{4}$ and using the identities in the Appendix properly, we find the following 9 equations between the constants:

$$
\begin{aligned}
w_{15}= & -\frac{3}{2}+2\left(w_{10}+w_{17}-w_{20}-w_{24}\right)-3\left(w_{29}-w_{30}-w_{6}\right)+w_{22}+w_{8}, \\
w_{13}= & \frac{2}{3}\left(w_{10}+w_{15}-w_{20}-w_{22}-w_{8}\right)+\frac{1}{3}\left(-w_{17}+w_{24}\right), \\
w_{21}= & \frac{2}{3}\left(w_{10}+w_{17}-w_{20}-w_{22}-w_{24}\right)+\frac{1}{2}\left(w_{12}-w_{27}-w_{7}\right)+\frac{1}{6} w_{15}-\frac{7}{6} w_{8}+w_{16}+w_{2}, \\
w_{26}= & -\frac{1}{3}\left(w_{8}-w_{15}\right)+\frac{2}{3}\left(-w_{10}+w_{20}+w_{22}\right)+\frac{8}{3}\left(-w_{17}+w_{24}\right)+6\left(w_{29}-w_{30}-w_{6}\right)+w_{14}-w_{16}+w_{19}+w_{27}-w_{7}, \\
w_{28}= & \frac{4}{3}\left(-w_{10}-w_{17}+w_{20}+w_{24}\right)+\frac{1}{3}\left(w_{8}-w_{15}+w_{22}\right)+4\left(w_{29}-w_{30}-w_{6}\right)+w_{27}-w_{7}, \\
w_{3}= & \frac{4}{3}\left(w_{10}-w_{20}\right)+\frac{1}{3}\left(w_{15}+w_{17}-w_{22}-w_{24}-w_{8}\right)-w_{29}+w_{30}+w_{6}, \quad w_{17}-w_{24}-w_{29}+w_{30}+w_{6}, \\
w_{5}= & \frac{2}{3}\left(w_{10}-w_{20}-w_{8}\right)+\frac{1}{2}\left(w_{14}+w_{29}-w_{30}-w_{6}-w_{7}\right)+\frac{1}{6}\left(w_{15}+w_{17}-w_{22}-w_{24}\right)-2 w_{1}-w_{18}+w_{2}, \\
w_{9}= & \frac{1}{6}\left(-w_{10}-w_{15}-w_{17}+w_{20}+w_{22}+w_{24}+w_{8}\right) .
\end{aligned}
$$


The nonzero coefficients could be fixed as follows by comparing the result with the action (4):

$w_{3}=w_{4}=w_{5}=w_{6}=\frac{1}{2}, \quad w_{7}=-\frac{3}{2}, \quad w_{27}=\frac{3}{2}, \quad w_{28}=1$.

By substituting these nonzero coefficients in (30), we can find the $S L(2, R)$ invariant form of the action (4).

$$
\begin{aligned}
L_{(\partial F)^{4}}^{S}= & \frac{1}{2} W_{b e f}{ }^{c a b} W_{d c}{ }^{f e}{ }_{a}^{d}+\frac{1}{2} W_{a}^{a b}{ }_{c b}^{c} W_{d}{ }^{d e}{ }_{f e}^{f} \\
& +\frac{1}{2} W_{b c}{ }^{f c a b} W_{d e f}{ }^{e}{ }_{a}^{d}+\frac{1}{2} W_{c d f}{ }^{c a b} W_{e b}{ }^{f e}{ }_{a}{ }^{d} \\
& -\frac{3}{2} W_{a}^{a b}{ }_{c d}{ }^{e} W^{d}{ }_{b}{ }^{c}{ }_{f e}^{f}+\frac{3}{2} W_{b e f}{ }^{c a b} W^{d}{ }_{a c}{ }^{f}{ }_{d}^{e} \\
& +W_{a}^{a b}{ }_{c e f} W^{d}{ }_{b}{ }^{c f}{ }_{d}^{e} .
\end{aligned}
$$

In order to reach the action at the order of six gauge fields and four derivatives, one should consider the expansion of the field $G^{\prime}$ up to the level of three gauge fields $F^{3}$. So the expansion of $\partial G^{\prime}$ up to this level could be found as follows:

$$
\begin{aligned}
\partial_{a} G_{b c}^{\prime}= & e^{-\phi_{0}} \partial_{a} F_{b c}+e^{-2 \phi_{0}}\left(\frac{1}{4} F_{d e} F^{d e} \partial_{a} F_{b c}\right. \\
& +F_{c}{ }^{d} F_{d}{ }^{e} \partial_{a} F_{b e}-F_{b}{ }^{d} F_{d}{ }^{e} \partial_{a} F_{c e} \\
& \left.-F_{b}{ }^{d} F_{c}{ }^{e} \partial_{a} F_{d e}+\frac{1}{2} F_{b c} F^{d e} \partial_{a} F_{d e}\right)+\cdots
\end{aligned}
$$

where dots refer to the terms at a higher order of gauge field.

Inserting the above expansion into the nonzero invariant structures $W W$ in (31), one can find the action containing six gauge fields with four derivatives that is compatible with $S$-duality. This action could appear in the simple form when one considering the total derivative terms. Actually, at the level of six gauge fields and four derivatives, there are 584 contractions with the structure $F F \partial F \partial F \partial F \partial F$. To find the total derivative terms, we note that there are 724 total derivative terms with the structure $\partial[F F F \partial F \partial F \partial F]$. Using their coefficients to eliminate the terms with the structure $F F F \partial \partial F \partial F \partial F$, one finds 483 constraint equations. Applying these constraints, the total derivative terms with the structure $F F \partial F \partial F \partial F \partial F$ could be found. Considering these terms and using the on-shell condition $\partial_{a} F^{a b}=0$, one can find the action corresponding to six gauge fields with four derivatives as follows in the simple form:

$$
\begin{aligned}
& L_{F^{2}(\partial F)^{4}}^{S} \sim \frac{3}{2} F^{a b} F^{c d} \partial_{c} F_{e}^{f} \partial_{d} F^{h i} \partial^{e} F_{a b} \partial_{f} F_{h i}+\frac{1}{4} F^{a b} F^{c d} \partial_{d} F_{c}^{f} \partial_{e} F^{h i} \partial^{e} F_{a b} \partial_{f} F_{h i}-3 F^{a b} F^{c d} \partial_{b} F_{e}^{f} \partial_{d} F^{h i} \partial^{e} F_{a c} \partial_{f} F_{h i} \\
& -\frac{1}{2} F^{a b} F^{c d} \partial_{d} F_{b}{ }^{f} \partial_{e} F^{h i} \partial^{e} F_{a c} \partial_{f} F_{h i}+3 F_{a}{ }^{c} F^{a b} \partial_{c} F^{h i} \partial_{d} F_{e}{ }^{f} \partial^{e} F_{b}{ }^{d} \partial_{f} F_{h i}-3 F_{a}{ }^{c} F^{a b} \partial_{c} F_{e}{ }^{f} \partial_{d} F^{h i} \partial^{e} F_{b}{ }^{d} \partial_{f} F_{h i} \\
& +\frac{1}{2} F_{a}{ }^{c} F^{a b} \partial_{c} F_{d}^{f} \partial_{e} F^{h i} \partial^{e} F_{b}{ }^{d} \partial_{f} F_{h i}-\frac{1}{2} F^{a b} F^{c d} \partial_{d} F^{h i} \partial^{e} F_{a c} \partial_{f} F_{h i} \partial^{f} F_{b e}+\frac{1}{4} F^{a b} F^{c d} \partial_{d} F^{h i} \partial^{e} F_{a b} \partial_{f} F_{h i} \partial^{f} F_{c e} \\
& -F_{a}{ }^{c} F^{a b} \partial_{d} F^{h i} \partial^{e} F_{b}{ }^{d} \partial_{f} F_{h i} \partial^{f} F_{c e}+\frac{1}{2} F_{a}{ }^{c} F^{a b} \partial_{c} F^{h i} \partial^{e} F_{b}{ }^{d} \partial_{f} F_{h i} \partial^{f} F_{d e}+F^{a b} F^{c d} \partial_{b} F_{a c} \partial_{e} F^{h i} \partial_{f} F_{h i} \partial^{f} F_{d}{ }^{e} \\
& -\frac{1}{2} F^{a b} F^{c d} \partial_{c} F_{a b} \partial_{e} F^{h i} \partial_{f} F_{h i} \partial^{f} F_{d}^{e}+F_{a}{ }^{c} F^{a b} \partial_{c} F_{b}{ }^{d} \partial_{e} F^{h i} \partial_{f} F_{h i} \partial^{f} F_{d}^{e}+3 F^{a b} F^{c d} \partial_{d} F_{h i} \partial_{e} F_{f}{ }^{i} \partial^{e} F_{a c} \partial^{h} F_{b}{ }^{f} \\
& +3 F^{a b} F^{c d} \partial_{d} F_{f i} \partial^{e} F_{a c} \partial_{h} F_{e}^{i} \partial^{h} F_{b}^{f}-2 F^{a b} F^{c d} \partial_{d} F_{e}{ }^{i} \partial^{e} F_{a c} \partial_{h} F_{f i} \partial^{h} F_{b}^{f}-\frac{3}{2} F^{a b} F^{c d} \partial_{d} F_{h i} \partial_{e} F_{f}{ }^{i} \partial^{e} F_{a b} \partial^{h} F_{c}^{f} \\
& +3 F_{a}{ }^{c} F^{a b} \partial_{e} F_{h i} \partial^{e} F_{b}{ }^{d} \partial_{f} F_{d}{ }^{i} \partial^{h} F_{c}{ }^{f}-2 F_{a}{ }^{c} F^{a b} \partial_{e} F_{d}{ }^{i} \partial^{e} F_{b}{ }^{d} \partial_{f} F_{h i} \partial^{h} F_{c}{ }^{f}+3 F_{a}{ }^{c} F^{a b} \partial^{e} F_{b}{ }^{d} \partial_{f} F_{e i} \partial_{h} F_{d}{ }^{i} \partial^{h} F_{c}{ }^{f} \\
& -\frac{3}{2} F^{a b} F^{c d} \partial_{d} F_{f i} \partial^{e} F_{a b} \partial_{h} F_{e}{ }^{i} \partial^{h} F_{c}{ }^{f}+F^{a b} F^{c d} \partial_{d} F_{e}{ }^{i} \partial^{e} F_{a b} \partial_{h} F_{f i} \partial^{h} F_{c}{ }^{f}-4 F_{a}{ }^{c} F^{a b} \partial_{e} F_{d}{ }^{i} \partial^{e} F_{b}{ }^{d} \partial_{h} F_{f i} \partial^{h} F_{c}{ }^{f} \\
& -3 F_{a}{ }^{c} F^{a b} \partial_{c} F_{h i} \partial_{e} F_{f}{ }^{i} \partial^{e} F_{b}{ }^{d} \partial^{h} F_{d}{ }^{f}-3 F_{a}{ }^{c} F^{a b} \partial_{c} F_{f i} \partial^{e} F_{b}{ }^{d} \partial_{h} F_{e}{ }^{i} \partial^{h} F_{d}{ }^{f}+2 F_{a}{ }^{c} F^{a b} \partial_{c} F_{e}{ }^{i} \partial^{e} F_{b}{ }^{d} \partial_{h} F_{f i} \partial^{h} F_{d}{ }^{f} \\
& +2 F^{a b} F^{c d} \partial_{c} F_{f}^{i} \partial_{d} F_{h i} \partial^{e} F_{a b} \partial^{h} F_{e}^{f}-4 F^{a b} F^{c d} \partial_{b} F_{f}{ }^{i} \partial_{d} F_{h i} \partial^{e} F_{a c} \partial^{h} F_{e}{ }^{f}+4 F_{a}^{c} F^{a b} \partial_{c} F_{h i} \partial_{d} F_{f}{ }^{i} \partial^{e} F_{b}{ }^{d} \partial^{h} F_{e}{ }^{f} \\
& -4 F_{a}{ }^{c} F^{a b} \partial_{c} F_{f}{ }^{i} \partial_{d} F_{h i} \partial^{e} F_{b}{ }^{d} \partial^{h} F_{e}{ }^{f}-\frac{3}{2} F_{a b} F^{a b} \partial_{e} F_{f i} \partial^{e} F^{c d} \partial^{h} F_{c}{ }^{f} \partial^{i} F_{d h}+2 F_{a b} F^{a b} \partial^{e} F^{c d} \partial_{f} F_{e i} \partial^{h} F_{c}{ }^{f} \partial^{i} F_{d h} \\
& +2 F_{a}{ }^{c} F^{a b} \partial_{e} F_{c}{ }^{f} \partial^{e} F_{b}{ }^{d} \partial_{f} F_{h i} \partial^{i} F_{d}^{h}+\frac{1}{2} F_{a b} F^{a b} \partial_{e} F_{c}{ }^{f} \partial^{e} F^{c d} \partial_{h} F_{f i} \partial^{i} F_{d}^{h}+2 F^{a b} F^{c d} \partial_{e} F_{c}{ }^{f} \partial^{e} F_{a b} \partial_{i} F_{f h} \partial^{i} F_{d}{ }^{h} \\
& -4 F^{a b} F^{c d} \partial_{e} F_{b}{ }^{f} \partial^{e} F_{a c} \partial_{i} F_{f h} \partial^{i} F_{d}{ }^{h}-4 F_{a}{ }^{c} F^{a b} \partial_{e} F_{c}{ }^{f} \partial^{e} F_{b}{ }^{d} \partial_{i} F_{f h} \partial^{i} F_{d}{ }^{h}+F_{a b} F^{a b} \partial_{e} F_{c}{ }^{f} \partial^{e} F^{c d} \partial_{i} F_{f h} \partial^{i} F_{d}{ }^{h} \\
& -2 F_{a}{ }^{c} F^{a b} \partial^{e} F_{b}{ }^{d} \partial^{f} F_{c e} \partial_{i} F_{f h} \partial^{i} F_{d}{ }^{h}+F_{a b} F^{a b} \partial^{e} F^{c d} \partial^{f} F_{c e} \partial_{i} F_{f h} \partial^{i} F_{d}^{h}+3 F^{a b} F^{c d} \partial_{b} F_{a c} \partial_{d} F^{e f} \partial_{f} F_{h i} \partial^{i} F_{e}{ }^{h}
\end{aligned}
$$




$$
\begin{aligned}
& -\frac{3}{2} F^{a b} F^{c d} \partial_{c} F_{a b} \partial_{d} F^{e f} \partial_{f} F_{h i} \partial^{i} F_{e}{ }^{h}+3 F_{a}{ }^{c} F^{a b} \partial_{c} F_{b}{ }^{d} \partial_{d} F^{e f} \partial_{f} F_{h i} \partial^{i} F_{e}{ }^{h}-\frac{1}{4} F_{a b} F^{a b} \partial^{e} F^{c d} \partial_{f} F_{h i} \partial^{f} F_{c d} \partial^{i} F_{e}{ }^{h} \\
& +3 F^{a b} F^{c d} \partial_{b} F_{a c} \partial_{f} F_{h i} \partial^{f} F_{d}{ }^{e} \partial^{i} F_{e}{ }^{h}-\frac{3}{2} F^{a b} F^{c d} \partial_{c} F_{a b} \partial_{f} F_{h i} \partial^{f} F_{d}^{e} \partial^{i} F_{e}{ }^{h}+3 F_{a}{ }^{c} F^{a b} \partial_{c} F_{b}{ }^{d} \partial_{f} F_{h i} \partial^{f} F_{d}{ }^{e} \partial^{i} F_{e}{ }^{h} \\
& +\frac{3}{4} F_{a b} F^{a b} \partial^{e} F^{c d} \partial^{f} F_{c d} \partial_{h} F_{f i} \partial^{i} F_{e}{ }^{h}+F^{a b} F^{c d} \partial_{d} F_{c}{ }^{f} \partial^{e} F_{a b} \partial_{i} F_{f h} \partial^{i} F_{e}{ }^{h}-2 F^{a b} F^{c d} \partial_{d} F_{b}{ }^{f} \partial^{e} F_{a c} \partial_{i} F_{f h} \partial^{i} F_{e}{ }^{h} \\
& +2 F_{a}{ }^{c} F^{a b} \partial_{c} F_{d}{ }^{f} \partial^{e} F_{b}{ }^{d} \partial_{i} F_{f h} \partial^{i} F_{e}{ }^{h}+F^{a b} F^{c d} \partial_{b} F_{a c} \partial^{f} F_{d}{ }^{e} \partial_{i} F_{f h} \partial^{i} F_{e}{ }^{h}-\frac{1}{2} F^{a b} F^{c d} \partial_{c} F_{a b} \partial^{f} F_{d}{ }^{e} \partial_{i} F_{f h} \partial^{i} F_{e}{ }^{h} \\
& +F_{a}{ }^{c} F^{a b} \partial_{c} F_{b}{ }^{d} \partial^{f} F_{d}{ }^{e} \partial_{i} F_{f h} \partial^{i} F_{e}{ }^{h}-F^{a b} F^{c d} \partial_{d} F_{h i} \partial_{e} F_{c}{ }^{f} \partial^{e} F_{a b} \partial^{i} F_{f}^{h}+2 F^{a b} F^{c d} \partial_{d} F_{h i} \partial_{e} F_{b}{ }^{f} \partial^{e} F_{a c} \partial^{i} F_{f}{ }^{h} \\
& -2 F_{a}{ }^{c} F^{a b} \partial_{c} F_{h i} \partial_{e} F_{d}{ }^{f} \partial^{e} F_{b}{ }^{d} \partial^{i} F_{f}^{h}-\frac{1}{4} F_{a b} F^{a b} \partial_{e} F_{c d} \partial^{e} F^{c d} \partial_{h} F_{f i} \partial^{i} F^{f h}-\frac{1}{2} F^{a b} F^{c d} \partial_{d} F_{c e} \partial^{e} F_{a b} \partial_{i} F_{f h} \partial^{i} F^{f h} \\
& -\frac{1}{2} F^{a b} F^{c d} \partial_{e} F_{c d} \partial^{e} F_{a b} \partial_{i} F_{f h} \partial^{i} F^{f h}+F^{a b} F^{c d} \partial_{d} F_{b e} \partial^{e} F_{a c} \partial_{i} F_{f h} \partial^{i} F^{f h}+F^{a b} F^{c d} \partial_{e} F_{b d} \partial^{e} F_{a c} \partial_{i} F_{f h} \partial^{i} F^{f h} \\
& -F_{a}{ }^{c} F^{a b} \partial_{c} F_{d e} \partial^{e} F_{b}{ }^{d} \partial_{i} F_{f h} \partial^{i} F^{f h}+F_{a}{ }^{c} F^{a b} \partial_{d} F_{c e} \partial^{e} F_{b}{ }^{d} \partial_{i} F_{f h} \partial^{i} F^{f h}+2 F_{a}{ }^{c} F^{a b} \partial_{e} F_{c d} \partial^{e} F_{b}{ }^{d} \partial_{i} F_{f h} \partial^{i} F^{f h} \\
& -\frac{1}{4} F_{a b} F^{a b} \partial_{e} F_{c d} \partial^{e} F^{c d} \partial_{i} F_{f h} \partial^{i} F^{f h}
\end{aligned}
$$

Because of the presence of the nonlinear $S L(2, R)$ invariant structures in the final $S$-dual form of the action (31), one can derive new couplings at the order of $\mathrm{N}$ gauge fields with four derivatives by expanding the invariant structures. We see that the consistency of the action (4) with the $S$-duality predicts the couplings (31) and the couplings at the level of more gauge fields in the Einstein frame. It would be interesting to confirm these couplings by direct calculations. $^{5}$

\section{ACKNOWLEDGMENTS}

The authors would like to kindly thank M. R. Garousi and A. A. Tseytlin for useful comments and discussions on related topics.

\section{APPENDIX: SOME IDENTITIES}

In this appendix we are going to find all identities that should be taken into account when one wants to write the action (4) in terms of $S L(2, R)$ invariant structures $W W$. In fact, the terms containing four dual gauge fields and four derivatives should be written in terms of four gauge fields and four derivatives.

Let us begin with the simple case, where we have four dual gauge fields with zero derivatives. There are two different contracted forms for these fields as $\tilde{F}_{a}^{c} \tilde{F}^{a b} \tilde{F}_{b}{ }^{d} \tilde{F}_{c d}$ and $\tilde{F}_{a b} \tilde{F}^{a b} \tilde{F}_{c d} \tilde{F}^{c d}$. Using the identity (19) one can write any of these terms in terms of four gauge field contractions $\left(\tilde{F}_{1} \tilde{F}_{2} \tilde{F}_{3} \tilde{F}_{4} \rightarrow \epsilon_{1} \epsilon_{2} \epsilon_{3} \epsilon_{4} F_{1} F_{2} F_{3} F_{4}\right)$. The result in this case is not dependent on the Levi-Civita pairing choices $\mathcal{E}, \mathcal{E}^{\prime}$, and $\mathcal{E}^{\prime \prime}$.

$$
\begin{aligned}
\tilde{F}_{a}{ }^{c} \tilde{F}^{a b} \tilde{F}_{b}{ }^{d} \tilde{F}_{c d} \rightarrow F_{a}{ }^{c} F^{a b} F_{b}{ }^{d} F_{c d}, \\
\tilde{F}_{a b} \tilde{F}^{a b} \tilde{F}_{c d} \tilde{F}^{c d} \rightarrow F_{a b} F^{a b} F_{c d} F^{c d} .
\end{aligned}
$$

In the next case, where we have four dual gauge fields and two derivatives, the result is dependent on the LeviCivita pairing choices. Consider all contractions of this case with arbitrary coefficients $y_{i}$ as follows:

$$
\begin{aligned}
& y_{1} \tilde{F}^{a b} \tilde{F}^{c d} \partial_{b} \tilde{F}_{d e} \partial_{c} \tilde{F}_{a}{ }^{e}+y_{2} \tilde{F}_{a}^{c} \tilde{F}^{a b} \partial_{b} \tilde{F}^{d e} \partial_{c} \tilde{F}_{d e}+y_{3} \tilde{F}^{a b} \tilde{F}^{c d} \partial_{c} \tilde{F}_{a}^{e} \partial_{d} \tilde{F}_{b e}+y_{4} \tilde{F}^{a b} \tilde{F}^{c d} \partial_{b} \tilde{F}_{a}^{e} \partial_{d} \tilde{F}_{c e}+y_{5} \tilde{F}_{a}{ }^{c} \tilde{F}^{a b} \partial_{d} \tilde{F}_{b}{ }^{d} \partial_{e} \tilde{F}_{c}{ }^{e} \\
& \quad+y_{6} \tilde{F}^{a b} \tilde{F}^{c d} \partial_{b} \tilde{F}_{a c} \partial_{e} \tilde{F}_{d}^{e}+y_{7} \tilde{F}^{a b} \tilde{F}^{c d} \partial_{c} \tilde{F}_{a b} \partial_{e} \tilde{F}_{d}^{e}+y_{8} \tilde{F}_{a}{ }^{c} \tilde{F}^{a b} \partial_{c} \tilde{F}_{b}{ }^{d} \partial_{e} \tilde{F}_{d}{ }^{e}+y_{9} \tilde{F}_{a b} \tilde{F}^{a b} \partial_{c} \tilde{F}^{c d} \partial_{e} \tilde{F}_{d}{ }^{e} \\
& \quad+y_{10} \tilde{F}^{a b} \tilde{F}^{c d} \partial_{d} \tilde{F}_{c e} \partial^{e} \tilde{F}_{a b}+y_{11} \tilde{F}^{a b} \tilde{F}^{c d} \partial_{e} \tilde{F}_{c d} \partial^{e} \tilde{F}_{a b}+y_{12} \tilde{F}^{a b} \tilde{F}^{c d} \partial_{d} \tilde{F}_{b e} \partial^{e} \tilde{F}_{a c}+y_{13} \tilde{F}^{a b} \tilde{F}^{c d} \partial_{e} \tilde{F}_{b d} \partial^{e} \tilde{F}_{a c} \\
& \quad+y_{14} \tilde{F}_{a}{ }^{c} \tilde{F}^{a b} \partial_{c} \tilde{F}_{d e} \partial^{e} \tilde{F}_{b}{ }^{d}+y_{15} \tilde{F}_{a}{ }^{c} \tilde{F}^{a b} \partial_{d} \tilde{F}_{c e} \partial^{e} \tilde{F}_{b}{ }^{d}+y_{16} \tilde{F}_{a}^{c} \tilde{F}^{a b} \partial_{e} \tilde{F}_{c d} \partial^{e} \tilde{F}_{b}{ }^{d}+y_{17} \tilde{F}_{a b} \tilde{F}^{a b} \partial_{d} \tilde{F}_{c e} \partial^{e} \tilde{F}^{c d} \\
& \quad+y_{18} \tilde{F}_{a b} \tilde{F}^{a b} \partial_{e} \tilde{F}_{c d} \partial^{e} \tilde{F}^{c d} .
\end{aligned}
$$

\footnotetext{
${ }^{5}$ The $S$-matrix elements may be constrained by both manifest Lorentz and duality invariance. Considering the duality invariance, as we did in this paper, may be interpreted as an intermediate step towards an analysis of the scattering amplitude [18].
} 
By selecting the Levi-Civita pairing choices $\mathcal{E}, \mathcal{E}^{\prime}$, and $\mathcal{E}^{\prime \prime}$, one can find the first term of above dual gauge content in terms of the following different gauge field combinations, respectively:

$$
\begin{aligned}
- & \frac{1}{4} F^{b c} F^{d e} \partial_{a} F_{d e} \partial^{a} F_{b c}+F^{b c} F^{d e} \partial^{a} F_{b c} \partial_{e} F_{d a}-F_{b}{ }^{d} F^{b c} \partial^{a} F_{c}{ }^{e} \partial_{e} F_{d a}=Y_{1}, \\
- & 2 F^{a b} F^{c d} \partial_{b} F_{a c} \partial_{e} F_{d}{ }^{e}+F^{a b} F^{c d} \partial_{c} F_{a b} \partial_{e} F_{d}{ }^{e}+\frac{1}{4} F^{a b} F^{c d} \partial_{e} F_{c d} \partial^{e} F_{a b}+2 F^{a b} F^{c d} \partial_{d} F_{b e} \partial^{e} F_{a c} \\
& -F^{a b} F^{c d} \partial_{e} F_{b d} \partial^{e} F_{a c}-F_{a}{ }^{c} F^{a b} \partial_{d} F_{c e} \partial^{e} F_{b}{ }^{d}=Y_{1}^{\prime}, \\
- & \frac{1}{2} F_{a}{ }^{c} F^{a b} \partial_{b} F^{e d} \partial_{c} F_{e d}-2 F_{a}{ }^{c} F^{a b} \partial_{c} F_{e d} \partial^{d} F_{b}{ }^{e}-F_{a}{ }^{c} F^{a b} \partial_{d} F_{c e} \partial^{d} F_{b}{ }^{e}+\frac{1}{4} F_{a b} F^{a b} \partial_{d} F_{c e} \partial^{d} F^{c e}+F^{a b} F^{c e} \partial_{b} F_{a}{ }^{d} \partial_{e} F_{c d} \\
& -\frac{1}{2} F_{a b} F^{a b} \partial^{d} F^{c e} \partial_{e} F_{c d}=Y_{1}^{\prime \prime} .
\end{aligned}
$$

As $Y_{1}, Y_{1}^{\prime}$, and $Y_{1}^{\prime \prime}$ are the expressions for a term containing dual gauge fields in terms of the gauge fields, they should be equal to each other. From $Y_{1}=Y_{1}^{\prime}=Y_{1}^{\prime \prime}$, one can find the following independent identities:

$$
\begin{aligned}
0= & 4 F^{a b} F^{c d} \partial_{b} F_{a c} \partial_{e} F_{d}{ }^{e}-2 F^{a b} F^{c d} \partial_{c} F_{a b} \partial_{e} F_{d}^{e}+2 F^{a b} F^{c d} \partial_{d} F_{c e} \partial^{e} F_{a b}-F^{a b} F^{c d} \partial_{e} F_{c d} \partial^{e} F_{a b}-4 F^{a b} F^{c d} \partial_{d} F_{b e} \partial^{e} F_{a c} \\
& +2 F^{a b} F^{c d} \partial_{e} F_{b d} \partial^{e} F_{a c}, \\
0= & 2 F_{a}{ }^{c} F^{a b} \partial_{b} F^{d e} \partial_{c} F_{d e}-4 F^{a b} F^{c d} \partial_{b} F_{a}{ }^{e} \partial_{d} F_{c e}+4 F^{a b} F^{c d} \partial_{d} F_{c e} \partial^{e} F_{a b}-F^{a b} F^{c d} \partial_{e} F_{c d} \partial^{e} F_{a b}+8 F_{a}{ }^{c} F^{a b} \partial_{c} F_{d e} \partial^{e} F_{b}{ }^{d} \\
& -4 F_{a}{ }^{c} F^{a b} \partial_{d} F_{c e} \partial^{e} F_{b}{ }^{d}+4 F_{a}{ }^{c} F^{a b} \partial_{e} F_{c d} \partial^{e} F_{b}{ }^{d}+2 F_{a b} F^{a b} \partial_{d} F_{c e} \partial^{e} F^{c d}-F_{a b} F^{a b} \partial_{e} F_{c d} \partial^{e} F^{c d} .
\end{aligned}
$$

To write the second term in (A1) in terms of the gauge fields, one finds three expressions $Y_{2}, Y_{2}^{\prime}$, and $Y_{2}^{\prime \prime}$ according to the Levi-Civita pairing choices $\mathcal{E}, \mathcal{E}^{\prime}$, and $\mathcal{E}^{\prime \prime}$. Considering the relation $Y_{2}=Y_{2}^{\prime}=Y_{2}^{\prime \prime}$ and the identities (A3), the another independent identity appears as follows:

$$
\begin{aligned}
0= & 4 F^{a b} F^{c d} \partial_{b} F_{d e} \partial_{c} F_{a}{ }^{e}+2 F_{a}{ }^{c} F^{a b} \partial_{b} F^{d e} \partial_{c} F_{d e}-4 F_{a}{ }^{c} F^{a b} \partial_{d} F_{b}{ }^{d} \partial_{e} F_{c}{ }^{e}-8 F^{a b} F^{c d} \partial_{b} F_{a c} \partial_{e} F_{d}{ }^{e}+8 F_{a}{ }^{c} F^{a b} \partial_{c} F_{b}{ }^{d} \partial_{e} F_{d}{ }^{e} \\
& -2 F_{a b} F^{a b} \partial_{c} F^{c d} \partial_{e} F_{d}{ }^{e}-4 F^{a b} F^{c d} \partial_{d} F_{c e} \partial^{e} F_{a b}+F^{a b} F^{c d} \partial_{e} F_{c d} \partial^{e} F_{a b}+8 F^{a b} F^{c d} \partial_{d} F_{b e} \partial^{e} F_{a c}-4 F^{a b} F^{c d} \partial_{e} F_{b d} \partial^{e} F_{a c} \\
& +4 F_{a}{ }^{c} F^{a b} \partial_{e} F_{c d} \partial^{e} F_{b}{ }^{d}-F_{a b} F^{a b} \partial_{e} F_{c d} \partial^{e} F^{c d} .
\end{aligned}
$$

Doing the same procedure for the third term in (A1) led to the following identity that is independent of previous identities:

$$
\begin{aligned}
0= & 2 F^{a c} F^{d e} \partial_{d} F_{a}{ }^{b} \partial_{e} F_{c b}-2 F_{a}{ }^{d} F^{a c} \partial_{e} F_{c}{ }^{e} \partial_{b} F_{d}{ }^{b}-2 F^{a c} F^{d e} \partial_{c} F_{a d} \partial_{b} F_{e}{ }^{b}-F^{a c} F^{d e} \partial_{d} F_{a c} \partial_{b} F_{e}{ }^{b}+4 F_{a}{ }^{d} F^{a c} \partial_{d} F_{c}{ }^{e} \partial_{b} F_{e}{ }^{b} \\
& -F_{a c} F^{a c} \partial_{d} F^{d e} \partial_{b} F_{e}{ }^{b}-F^{a c} F^{d e} \partial_{e} F_{d b} \partial^{b} F_{a c}-2 F^{a c} F^{d e} \partial_{e} F_{c b} \partial^{b} F_{a d}-4 F_{a}{ }^{d} F^{a c} \partial_{d} F_{e b} \partial^{b} F_{c}{ }^{e}+2 F_{a}{ }^{d} F^{a c} \partial_{e} F_{d b} \partial^{b} F_{c}{ }^{e} \\
& -F_{a c} F^{a c} \partial_{e} F_{d b} \partial^{b} F^{d e} .
\end{aligned}
$$

After finding the other terms in (A1) in terms of the gauge fields $\left(Y_{i}, Y_{i}^{\prime}\right.$, and $Y_{i}^{\prime \prime}$ where $\left.i=3, \ldots, 18\right)$, one finds that the relations $Y_{i}=Y_{i}^{\prime}=Y_{i}^{\prime \prime}$ do not produce any new independent identities.

Let us now consider the third case where we have four gauge fields and four derivatives. One can find all contracted forms of four dual gauge fields with four derivatives by using "xAct" as follows: 


$$
\begin{aligned}
& z_{1} \partial_{c} \tilde{F}^{e f} \partial^{c} \tilde{F}^{a b} \partial_{d} \tilde{F}_{e f} \partial^{d} \tilde{F}_{a b}+z_{2} \partial_{a} \tilde{F}^{a b} \partial_{c} \tilde{F}^{e f} \partial_{d} \tilde{F}_{e f} \partial^{d} \tilde{F}_{b}^{c}+z_{3} \partial_{b} \tilde{F}_{e f} \partial^{c} \tilde{F}^{a b} \partial_{d} \tilde{F}_{c}^{f} \partial^{e} \tilde{F}_{a}^{d}+z_{4} \partial_{b} \tilde{F}_{c}^{f} \partial^{c} \tilde{F}^{a b} \partial_{d} \tilde{F}_{e f} \partial^{e} \tilde{F}_{a}{ }^{d} \\
& +z_{5} \partial_{c} \tilde{F}_{d f} \partial^{c} \tilde{F}^{a b} \partial_{e} \tilde{F}_{b}^{f} \partial^{e} \tilde{F}_{a}^{d}+z_{6} \partial_{a} \tilde{F}^{a b} \partial_{c} \tilde{F}_{b}^{c} \partial_{d} \tilde{F}^{d e} \partial_{f} \tilde{F}_{e}^{f}+z_{7} \partial_{a} \tilde{F}^{a b} \partial_{c} \tilde{F}_{d}^{e} \partial^{d} \tilde{F}_{b}^{c} \partial_{f} \tilde{F}_{e}^{f} \\
& +z_{8} \partial_{a} \tilde{F}^{a b} \partial_{d} \tilde{F}_{c}{ }^{e} \partial^{d} \tilde{F}_{b}^{c} \partial_{f} \tilde{F}_{e}^{f}+z_{9} \partial_{a} \tilde{F}^{a b} \partial_{b} \tilde{F}^{c d} \partial^{e} \tilde{F}_{c d} \partial_{f} \tilde{F}_{e}^{f}+z_{10} \partial_{a} \tilde{F}^{a b} \partial^{d} \tilde{F}_{b}{ }^{c} \partial^{e} \tilde{F}_{c d} \partial_{f} \tilde{F}_{e}^{f} \\
& +z_{11} \partial^{c} \tilde{F}^{a b} \partial^{e} \tilde{F}_{a}{ }^{d} \partial_{f} \tilde{F}_{c e} \partial^{f} \tilde{F}_{b d}+z_{12} \partial_{c} \tilde{F}_{d f} \partial^{c} \tilde{F}^{a b} \partial^{e} \tilde{F}_{a}{ }^{d} \partial^{f} \tilde{F}_{b e}+z_{13} \partial^{c} \tilde{F}^{a b} \partial_{d} \tilde{F}_{c f} \partial^{e} \tilde{F}_{a}{ }^{d} \partial^{f} \tilde{F}_{b e} \\
& +z_{14} \partial^{c} \tilde{F}^{a b} \partial^{e} \tilde{F}_{a}{ }^{d} \partial_{f} \tilde{F}_{c d} \partial^{f} \tilde{F}_{b e}+z_{15} \partial^{c} \tilde{F}^{a b} \partial_{d} \tilde{F}_{e f} \partial^{d} \tilde{F}_{a c} \partial^{f} \tilde{F}_{b}{ }^{e}+z_{16} \partial_{c} \tilde{F}_{a}{ }^{d} \partial^{c} \tilde{F}^{a b} \partial_{e} \tilde{F}_{d f} \partial^{f} \tilde{F}_{b}{ }^{e} \\
& +z_{17} \partial^{c} \tilde{F}^{a b} \partial^{d} \tilde{F}_{a c} \partial_{e} \tilde{F}_{d f} \partial^{f} \tilde{F}_{b}{ }^{e}+z_{18} \partial_{c} \tilde{F}_{a}{ }^{d} \partial^{c} \tilde{F}^{a b} \partial_{f} \tilde{F}_{d e} \partial^{f} \tilde{F}_{b}{ }^{e}+z_{19} \partial^{c} \tilde{F}^{a b} \partial^{d} \tilde{F}_{a c} \partial_{f} \tilde{F}_{d e} \partial^{f} \tilde{F}_{b}{ }^{e} \\
& +z_{20} \partial_{a} \tilde{F}^{a b} \partial_{b} \tilde{F}^{c d} \partial_{d} \tilde{F}_{e f} \partial^{f} \tilde{F}_{c}{ }^{e}+z_{21} \partial^{c} \tilde{F}^{a b} \partial_{d} \tilde{F}_{e f} \partial^{d} \tilde{F}_{a b} \partial^{f} \tilde{F}_{c}{ }^{e}+z_{22} \partial_{a} \tilde{F}^{a b} \partial_{d} \tilde{F}_{e f} \partial^{d} \tilde{F}_{b}{ }^{c} \partial^{f} \tilde{F}_{c}{ }^{e} \\
& +z_{23} \partial^{c} \tilde{F}^{a b} \partial^{d} \tilde{F}_{a b} \partial_{e} \tilde{F}_{d f} \partial^{f} \tilde{F}_{c}{ }^{e}+z_{24} \partial_{a} \tilde{F}^{a b} \partial^{d} \tilde{F}_{b}{ }^{c} \partial_{e} \tilde{F}_{d f} \partial^{f} \tilde{F}_{c}{ }^{e}+z_{25} \partial^{c} \tilde{F}^{a b} \partial^{d} \tilde{F}_{a b} \partial_{f} \tilde{F}_{d e} \partial^{f} \tilde{F}_{c}{ }^{e} \\
& +z_{26} \partial_{a} \tilde{F}^{a b} \partial^{d} \tilde{F}_{b}{ }^{c} \partial_{f} \tilde{F}_{d e} \partial^{f} \tilde{F}_{c}{ }^{e}+z_{27} \partial_{b} \tilde{F}_{e f} \partial^{c} \tilde{F}^{a b} \partial^{d} \tilde{F}_{a c} \partial^{f} \tilde{F}_{d}^{e}+z_{28} \partial_{a} \tilde{F}^{a b} \partial_{c} \tilde{F}_{e f} \partial^{d} \tilde{F}_{b}^{c} \partial^{f} \tilde{F}_{d}^{e} \\
& +z_{29} \partial_{a} \tilde{F}^{a b} \partial_{c} \tilde{F}_{b}{ }^{c} \partial_{e} \tilde{F}_{d f} \partial^{f} \tilde{F}^{d e}+z_{30} \partial_{b} \tilde{F}_{a c} \partial^{c} \tilde{F}^{a b} \partial_{e} \tilde{F}_{d f} \partial^{f} \tilde{F}^{d e}+z_{31} \partial_{c} \tilde{F}_{a b} \partial^{c} \tilde{F}^{a b} \partial_{e} \tilde{F}_{d f} \partial^{f} \tilde{F}^{d e} \\
& +z_{32} \partial_{a} \tilde{F}^{a b} \partial_{c} \tilde{F}_{b}^{c} \partial_{f} \tilde{F}_{d e} \partial^{f} \tilde{F}^{d e}+z_{33} \partial_{c} \tilde{F}_{a b} \partial^{c} \tilde{F}^{a b} \partial_{f} \tilde{F}_{d e} \partial^{f} \tilde{F}^{d e},
\end{aligned}
$$

where $z_{i}$ are some unknown coefficients.

As in previous cases, we do the same procedure to find the above dual gauge fields in terms of relevant gauge fields. Different choices of Levi-Civita pairings led to different results that should be equal to each other: $Z_{i}=$ $Z_{i}^{\prime}=Z_{i}^{\prime \prime}$ and $i=1, \ldots, 33$. These equalities led to ten independent identities, three of which we present in the following. To investigate the $S$-duality behavior of amplitude and actions at the presence of a D-brane at the level of four gauge fields and four (/N) derivatives, one has to use these identities as we have done to write the action (4) in terms of the $S$-dual invariant structures (31).

$$
\begin{aligned}
0= & \partial_{c} F^{e f} \partial^{c} F^{a b} \partial_{d} F_{e f} \partial^{d} F_{a b}-2 \partial_{c} F_{d f} \partial^{c} F^{a b} \partial_{e} F_{b}{ }^{f} \partial^{e} F_{a}{ }^{d}-4 \partial^{c} F^{a b} \partial^{e} F_{a}{ }^{d} \partial_{f} F_{c e} \partial^{f} F_{b d}+8 \partial_{c} F_{d f} \partial^{c} F^{a b} \partial^{e} F_{a}{ }^{d} \partial^{f} F_{b e} \\
& +4 \partial^{c} F^{a b} \partial^{e} F_{a}{ }^{d} \partial_{f} F_{c d} \partial^{f} F_{b e}-4 \partial^{c} F^{a b} \partial_{d} F_{e f} \partial^{d} F_{a c} \partial^{f} F_{b}^{e}+4 \partial^{c} F^{a b} \partial_{d} F_{e f} \partial^{d} F_{a b} \partial^{f} F_{c}{ }^{e}, \\
0= & \partial_{c} F^{e f} \partial^{c} F^{a b} \partial_{d} F_{e f} \partial^{d} F_{a b}-4 \partial^{c} F^{a b} \partial_{d} F_{e f} \partial^{d} F_{a c} \partial^{f} F_{b}{ }^{e}+4 \partial_{c} F_{a}{ }^{d} \partial^{c} F^{a b} \partial_{e} F_{d f} \partial^{f} F_{b}{ }^{e}-4 \partial_{c} F_{a}{ }^{d} \partial^{c} F^{a b} \partial_{f} F_{d e} \partial^{f} F_{b}{ }^{e} \\
& +8 \partial^{c} F^{a b} \partial^{d} F_{a c} \partial_{f} F_{d e} \partial^{f} F_{b}{ }^{e}+4 \partial^{c} F^{a b} \partial_{d} F_{e f} \partial^{d} F_{a b} \partial^{f} F_{c}^{e}-2 \partial^{c} F^{a b} \partial^{d} F_{a b} \partial_{f} F_{d e} \partial^{f} F_{c}{ }^{e}-2 \partial_{c} F_{a b} \partial^{c} F^{a b} \partial_{e} F_{d f} \partial^{f} F^{d e} \\
& +\partial_{c} F_{a b} \partial^{c} F^{a b} \partial_{f} F_{d e} \partial^{f} F^{d e}, \\
0= & \partial_{c} F^{e f} \partial^{c} F^{a b} \partial_{d} F_{e f} \partial^{d} F_{a b}+4 \partial_{a} F^{a b} \partial_{c} F^{e f} \partial_{d} F_{e f} \partial^{d} F_{b}^{c}-4 \partial_{c} F_{d f} \partial^{c} F^{a b} \partial_{e} F_{b}^{f} \partial^{e} F_{a}{ }^{d}+8 \partial_{c} F_{d f} \partial^{c} F^{a b} \partial^{e} F_{a} \partial^{f} F_{b e} \\
& +4 \partial^{c} F^{a b} \partial_{d} F_{e f} \partial^{d} F_{a c} \partial^{f} F_{b}^{e}-4 \partial_{c} F_{a}{ }^{d} \partial^{c} F^{a b} \partial_{e} F_{d f} \partial^{f} F_{b}^{e}+4 \partial_{c} F_{a}{ }^{d} \partial^{c} F^{a b} \partial_{f} F_{d e} \partial^{f} F_{b}^{e}-8 \partial^{c} F^{a b} \partial^{d} F_{a c} \partial_{f} F_{d e} \partial^{f} F_{b}{ }^{e} \\
& +8 \partial_{a} F^{a b} \partial_{d} F_{e f} \partial^{d} F_{b}{ }^{c} \partial^{f} F_{c}{ }^{e}+2 \partial^{c} F^{a b} \partial^{d} F_{a b} \partial_{f} F_{d e} \partial^{f} F_{c}{ }^{e}+2 \partial_{c} F_{a b} \partial^{c} F^{a b} \partial_{e} F_{d f} \partial^{f} F^{d e}-\partial_{c} F_{a b} \partial^{c} F^{a b} \partial_{f} F_{d e} \partial^{f} F^{d e} .
\end{aligned}
$$

These identities and the other ones that appear in this section could be checked explicitly by replacing $F_{a b}=\partial_{a} A_{b}-\partial_{b} A_{a}$ (or by substituting the component of $F_{a b}$ ).

[1] M. K. Gaillard and B. Zumino, Nucl. Phys. B193, 221 (1981).

[2] K. Becker, M. Becker, and J. H. Schwarz, String Theory and M-Theory (Cambridge University Press, Cambridge, England, 2007), p. 739.

[3] M. B. Green and M. Gutperle, Phys. Lett. B 377, 28 (1996).

[4] K. B. Velni and H. Babaei-Aghbolagh, Nucl. Phys. B913, 987 (2016).

[5] J. Polchinski, Phys. Rev. Lett. 75, 4724 (1995).
[6] R. Roiban and A. A. Tseytlin, J. High Energy Phys. 10 (2012) 099.

[7] M. R. Garousi, Phys. Rep. 702, 1 (2017).

[8] M. R. Garousi, Phys. Rev. D 84, 126019 (1103).

[9] H. Babaei-Aghbolagh and M. R. Garousi, Phys. Rev. D 88, 026008 (2013); M. R. Garousi, Nucl. Phys. B862, 107 (2012).

[10] K. B. Velni, Phys. Rev. D 93, 066009 (2016); K. B. Velni and A. Jalali, Int. J. Mod. Phys. A 33, 1850106 (2018); 
S. Karimi and M. R. Garousi, Nucl. Phys. B939, 485 (2019); K. B. Velni and A. Jalali, Phys. Rev. D 95, 086010 (2017); 95, 026004 (2017).

[11] M. R. Garousi, Phys. Rev. D 93, 066014 (2016).

[12] A. Abouelsaood, C. G. Callan, Jr., C. R. Nappi, and S. A. Yost, Nucl. Phys. B280, 599 (1987).

[13] A. A. Tseytlin, Phys. Lett. B 202, 81 (1988).

[14] O. D. Andreev and A. A. Tseytlin, Nucl. Phys. B311, 205 (1988).

[15] N. Wyllard, Nucl. Phys. B598, 247 (2001).

[16] O. Andreev, Phys. Lett. B 513, 207 (2001).

[17] N. Wyllard, J. High Energy Phys. 08 (2001) 027.

[18] W. Chemissany, R. Kallosha, and T. Ortin, Phys. Rev. D 85, 046002 (2012); J. J. M. Carrascoa, R. Kallosha, and R. Roiban, Phys. Rev. D 85, 025007 (2012).

[19] W. A. Chemissany, J. de Jong, and M. de Roo, J. High Energy Phys. 11 (2006) 086.
[20] A. A. Tseytlin, Nucl. Phys. B469, 51 (1996).

[21] E. A. Bergshoeff, M. de Roo, S. F. Kerstan, T. Ortin, and F. Riccioni, J. High Energy Phys. 02 (2007) 007.

[22] E. Bergshoeff, P. S. Howe, S. Kerstan, and L. Wulff, J. High Energy Phys. 10 (2007) 050.

[23] M. Shmakova, Phys. Rev. D 62, 104009 (2000).

[24] G. W. Gibbons and D. A. Rasheed, Phys. Lett. B 365, 46 (1996).

[25] M. R. Garousi, J. High Energy Phys. 04 (2012) 140.

[26] E. Hatefi, J. High Energy Phys. 05 (2010) 080.

[27] A. De Giovanni, A. Santambrogio, and D. Zanon, Phys. Lett. B 472, 94 (2000).

[28] A. Hashimoto and I. R. Klebanov, Nucl. Phys. B, Proc. Suppl. 55B, 118 (1997).

[29] T. Nutma, Comput. Phys. Commun. 185, 1719 (2014); J. High Energy Phys. 08 (2011) 074. 